\title{
Super-resolution sensing with a randomly scattering analyzer
}

\author{
Qiaoen Luo, Justin A. Patel $\odot$, and Kevin J. Webb $\odot^{*}$ \\ Purdue University, West Lafayette, Indiana 47907-2035, USA
}

(Received 18 June 2021; accepted 9 November 2021; published 23 December 2021)

\begin{abstract}
A randomly scattering analyzer located in the far field and with a fixed aperture, in front of a multielement detector, is introduced as a means to access enhanced sensing information associated with far-subwavelength spatial features. This sensing method allows improved spatial resolution with coherent fields scattered from a moving object, or some other relative change that causes a modified field incident on the detector aperture. Experimental optical speckle correlation data with a translated diffusing structure show the salient features, and understanding in relation to the experimental variables is supported by numerical simulations. The conclusion is that more heavily scattering analyzers provide better spatial resolution because the measurements are more sensitive to changes in the incident field. Such randomly scattering analyzers offer a dimension for sensitive coherent optical metrology related to various sensing and motion application domains requiring large offset distances.
\end{abstract}

DOI: 10.1103/PhysRevResearch.3.L042045

The physics of disordered media is of profound importance in quantum transport and statistical optics [1], with broad ramifications that include the modeling of small-scale electronic devices [2], imaging through scattering media [3], and studies of localization [4]. Associated applications of random matrix theory have been insightful and have found lasting relevance because of their broad significance [2,5], including for controlling or accessing information through random channels [1,6]. Notably, wave-front regulation for imaging purposes is possible with measurement and application of the field transmission matrix [7,8]. However, despite the information that in principle exists in coherent waves that have passed through a randomly scattering medium, extraction or control remains challenging. Separately, the enormous practical consequences of exceeding Abbe's resolution limit, notably in the biosciences, have led to the substantial impact of various forms of super-resolution imaging utilizing either the point spread function of a microscope [9-11] or higher-order photon correlations using quantum information [12-15].

We introduce the concept of a randomly scattering analyzer, which enables super-resolution sensitivity to changes in the field incident upon it that could be associated with a variety of imaging or sensing contexts. Here, we focus on experiments involving the motion of a remote diffusely scattering object illuminated by a coherent incident field. However, the changes could be due to motion of a scatterer or set of scatterers, and could be the result of small deformations

\footnotetext{
*Corresponding author: webb@purdue.edu

Published by the American Physical Society under the terms of the Creative Commons Attribution 4.0 International license. Further distribution of this work must maintain attribution to the author(s) and the published article's title, journal citation, and DOI.
}

in a solid-state material. The random analyzer is shown to provide access to far-subwavelength spatial information with far-field measurement and a fixed aperture, thereby breaking the (far-field, wavelength-scale) diffraction limit on spatial resolution by a substantial margin. This enables a paradigm in sensing and broadens physical insight into wave transport in disordered media.

Our experimental arrangement is shown in Fig. 1, where a fixed randomly scattering analyzer slab is placed between the moving diffusing object of interest and the camera, all of which are arranged in the path of a fixed incident laser beam. A 59-mW, 850-nm laser diode with a linewidth less than $10 \mathrm{MHz}$ was used for illumination, producing a $0.4-$ mm-diameter laser beam spot incident upon the translated diffusing object. A linear computer-controlled stage was used to move the slab in subwavelength steps of $476 \mathrm{~nm}$. The scattering analyzers used were a single ground-glass slide of 1500 grit $(10 \mathrm{~cm} \times 10 \mathrm{~cm} \times 0.2 \mathrm{~cm})$, a 3 -mm-thick acrylic slab, and a 6-mm-thick acrylic slab. Both acrylic analyzer slabs $(14 \mathrm{~cm} \times 14 \mathrm{~cm})$ had a reduced scattering coefficient of $4 \mathrm{~cm}^{-1}$, with mean distance between the $50-\mathrm{nm} \mathrm{TiO}_{2}$ scattering centers being large relative to the wavelength [16]. An area of approximately $1.8 \mathrm{~mm} \times 1.8 \mathrm{~mm}$ on the back of the second scattering layer (the analyzer) was imaged by a Photometrics Prime sCMOS camera $(2048 \times 2048$ pixels $)$ using magnifying optics. The $4 \mathrm{~F}$ system with an aperture in the Fourier plane in Fig. 1 was used to regulate the size of the speckle at the camera, so that there is an adequate number of pixels within the correlation area and a sufficient number of correlation domains for averaging (possible because the statistics are stationary over the camera image). The speckle size at the camera is estimated to be consistent throughout our experiments: around 15 pixels within the full width at half maximum of the autocorrelation of the speckle patterns. Measurement through a linear (copolarized) polarizer yielded zero-mean circular Gaussian and the measured negative exponential intensity statistics. 


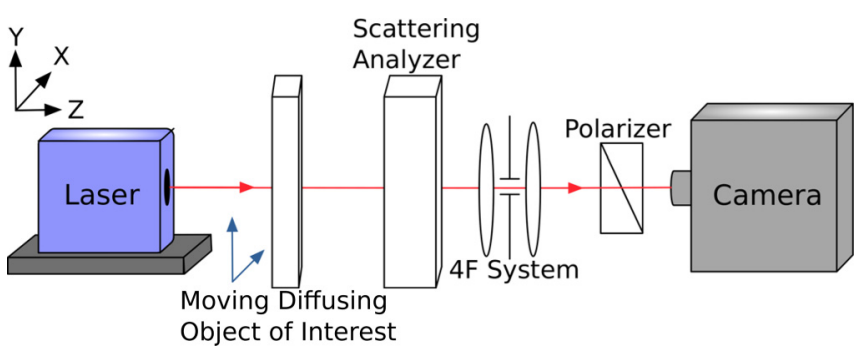

FIG. 1. Experimental arrangement, where a coherent laser illuminates a randomly scattering slab and speckle images are captured through a random analyzer. An area of approximately $1.8 \mathrm{~mm} \times$ $1.8 \mathrm{~mm}$ on the back of the random analyzer (the second scattering layer) was imaged (using a magnifying lens that is not shown) with a Photometrics Prime sCMOS $(2048 \times 2048$ pixels $)$ camera. The $4 \mathrm{~F}$ system was used to regulate the speckle size and hence the number of speckle spots on the camera (for adequate resolution and useful statistical averaging). The diffusing slab was translated in small steps, and a speckle pattern was captured at each position through a linear polarizer (with the same same polarization as the incident laser beam). The measured data is presented as intensity correlations of these speckle patterns as a function of the translated slab position.

Figure 2 shows the measured normalized average speckle intensity correlation as a function of translated diffuser position, $\langle\tilde{I}(x) \tilde{I}(x+\Delta x)\rangle$, with $\tilde{I}=(I-\langle I\rangle) /\langle I\rangle$ and $\langle I\rangle$ the mean intensity in the image, for varying degrees of scatter in the random analyzer, while otherwise keeping the experimental configuration the same. The translated diffuser was a $3-\mathrm{mm}$ thick acrylic slab containing $50-\mathrm{nm} \mathrm{TiO}_{2}$ scatterers having a reduced scattering coefficient of $4 \mathrm{~cm}^{-1}$ and negligible absorption [16]. The translated slab and the scattering analyzer were separated by a distance of about $5 \mathrm{~cm}$. This is our key and motivating result, because it shows that an increasing amount of scatter in the analyzer results in a more rapid decorrelation

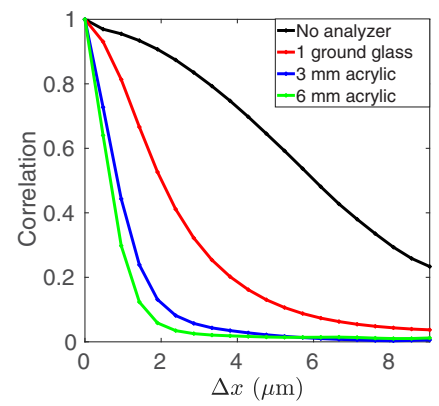

FIG. 2. The averaged normalized intensity correlation as a function of translation of the diffusing object (3-mm-thick acrylic slab containing 50-nm $\mathrm{TiO}_{2}$ scattering having a reduced scattering coefficient of $4 \mathrm{~cm}^{-1}$ ), showing a varying decorrelation rate for the different analyzers. The more scattering the analyzer is, the faster the decorrelation becomes. With a thicker analyzer, we are able to sense subwavelength $(<850 \mathrm{~nm})$ translation of the diffusing object. For $\Delta x=952 \mathrm{~nm}$, the measured intensity correlations were 0.9550 (no analyzer), 0.8134 (one ground-glass slide), 0.4431 (3-mm acrylic slab), and 0.2978 (6-mm acrylic slab). Both types of acrylic random analyzer (with $50-\mathrm{nm} \mathrm{TiO}_{2}$ scatterers) have a reduced scattering coefficient of $4 \mathrm{~cm}^{-1}$.

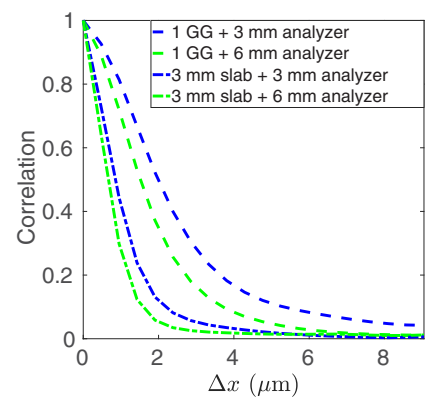

(a)

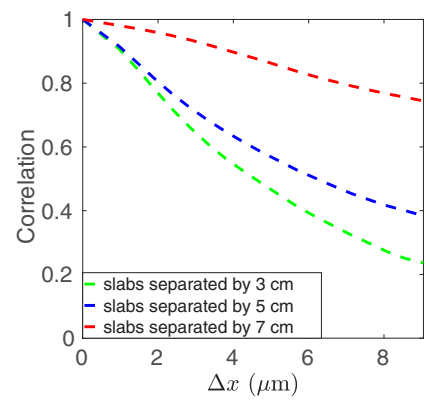

(b)
FIG. 3. Experimental speckle correlation data with additional variables. (a) The more-scattering 3-mm-thick acrylic slab produced faster decorrelation when compared to one moving ground-glass slide (1 GG), because the larger spread of speckle intensity exiting the acrylic slab results in smaller speckle spots incident on the analyzer. (b) The rate of speckle decorrelation increased for smaller separation between the diffusing moving object (one ground-glass slide) and the analyzer (3-mm-thick acrylic slab), because of the reducing speckle size.

and hence greater sensitivity to the changing incident field associated with the moving diffuser. Notice that, despite the large distance between the moving slab and the analyzer, farsubwavelength translation can be sensed. A subtle pattern in the experimental results displayed in Fig. 2 is the seemingly diminishing returns on the increase in the decorrelation rate that one sees by increasing the amount of scatter in the analyzer.

With reference to the arrangement in Fig. 1, we consider now our experimental results involving the influence of variations in the scattering strength of the moving diffuser [Fig. 3(a)] and the separation distance between the analyzer and the moving object [Fig. 3(b)]. Referring to Fig. 3(a), more scatter in the translated diffusing slab resulted in a faster intensity decorrelation. Also, Fig. 3(b) shows that the rate of speckle decorrelation increased for smaller separation between the diffusing moving object (1 ground-glass slide in this case) and the analyzer (3-mm-thick acrylic slab). Both phenomena can be understood as diffractive effects, based on light passing through the moving scattering slab, where the field at the analyzer is dictated (in the far field) by the Fourier transform of that on the detector side of the translated diffuser. With more scatter in the translated slab, the effective spatial support of the light transmitted through that slab (with a fixed and small laser spot on the source side, referring to Fig. 1) increases (as measured on the detector side of that slab). Through the Fourier transform, this spatial support regulates the speckle size at the analyzer, and increasing the spatial support (scatter) in the diffusing slab reduces the speckle size. This reduction of the speckle size at the analyzer results in more rapid decorrelation for the more heavily scattering translated diffuser in Fig. 3(a). Likewise, there is a geometrical scaling of speckle size with separation distance between the translated diffuser and the analyzer, with reduced separation producing smaller speckle at the analyzer and hence the more rapid decorrelation observed in Fig. 3(b). In the limit of a translated normally incident uniform plane wave, there would be no decorrelation (the infinite-speckle-size limit). Thus, our 


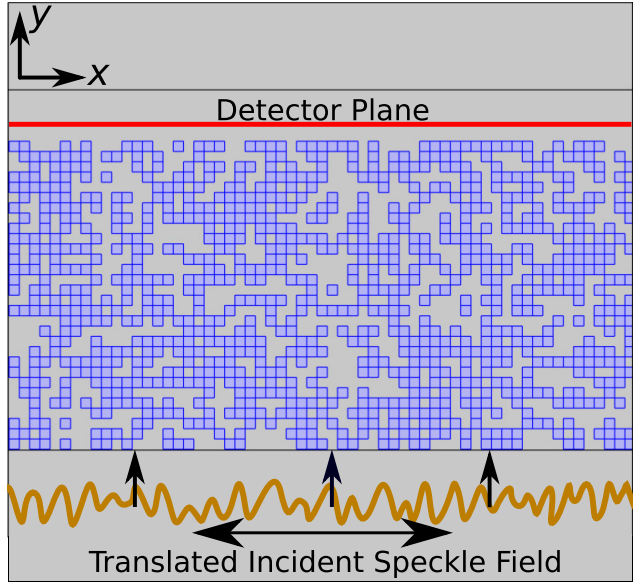

FIG. 4. The numerical simulation arrangement showing a section of one of the 6- $\mu \mathrm{m}$-thick random analyzer slabs (the full breadth of the slab is wider than the displayed section). The randomly populated blue squares have side lengths of $200 \mathrm{~nm}$ and consist of dielectric material, with a fill fraction of $50 \%$, while the gray regions are free space. The bottom and top rectangular sections are perfectly matched layers in a scattered field solution. A randomly generated speckle field (brown) was translated along the slab and in the $x$ direction. The speckle field had $E_{z}, H_{x}, H_{y}$ polarization, and the free-space wavelength was $\lambda=850 \mathrm{~nm}$. The $y$ component of the time-average Poynting vector was measured at the detector plane (red), a distance of $\lambda / 4$ from the top boundary of the scattering slab.

conclusion is that more scatter in the moving diffuser or smaller distance to the analyzer both result in smaller speckle size at the analyzer and hence more rapid decorrelation. Figure 3(b) again shows that increasing the amount of scatter in the analyzer increases sensitivity to motion or changes in the field incident on the analyzer.

While the experimental results in Figs. 2 and 3 have a compelling set of trends with regard to enhanced subwavelength spatial sensitivity, seeking deeper understanding leads us to numerical simulations. We present two-dimensional finite element method simulations involving translation of the random field incident on a scattering analyzer. These simulations capture the essence of the experiment (where the field incident on the analyzer was changed with a fixed laser beam illuminating a translated scattering slab) while providing a tractable computational problem. As visualized in Fig. 4, an analyzer slab region that has a thickness and breadth of $L_{y}$ and $L_{x}$, respectively, was pixelated and randomly populated (squares $200 \mathrm{~nm}$ on each side, fill fraction of 0.5 for all simulations) with a lossless dielectric material (relative permittivity $\epsilon_{r}$ ) to represent an appropriate level of analyzer scatter. All simulations used $L_{x}=60 \mu \mathrm{m}$, though $L_{y}$ varied between 3 or $6 \mu \mathrm{m}$. Transverse periodic boundary conditions were applied, with the width $(\Lambda)$ just slightly larger than $L_{x}\left(\Lambda=L_{x}+2 \lambda / 50\right.$, with $\lambda=850 \mathrm{~nm}$ the free-space wavelength), creating a discrete set of modes (plane waves) defining the incident field (in free space). Spatially random incident fields (TE with $E_{z}, H_{x}, H_{y}$, $\lambda=850 \mathrm{~nm}$ ) were generated in the (spatial) spectral domain from a zero-mean circular Gaussian density function, and the spatial correlation length (speckle size in the space domain) was regulated by the number of modes (with the maximum

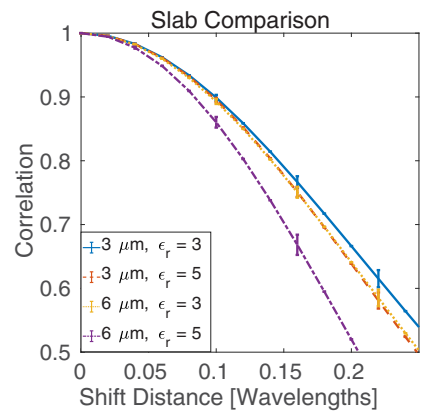

(a)

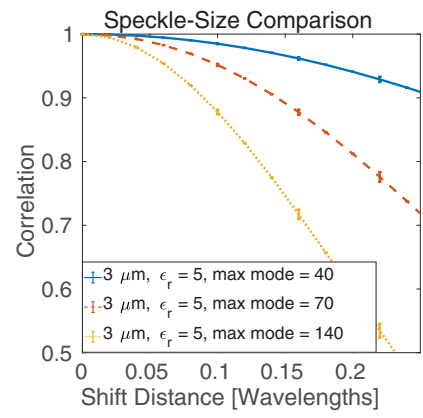

(b)
FIG. 5. Numerical simulations of the transmitted intensity correlation as a function of translated random incident fields, with $\lambda=$ $850 \mathrm{~nm}$, showing the influence of (a) random analyzer scatter level and (b) speckle size. The fields are zero-mean circular Gaussian. The error bars correspond to $\pm \sigma_{e}$, where $\sigma_{e}$ is the standard error in estimating the mean from the samples. Increasing the analyzer scatter and decreasing the speckle size both lead to more rapid decorrelation and hence greater sensitivity to spatial translation of the field.

number of modes yielding a speckle size of about half a wavelength, measured as the full width at half maximum of the speckle intensity autocorrelation). Transmitted field intensities (defined by the time-average Poynting vector magnitude in the $y$ direction) were determined at a distance of $\lambda / 4$ away from the back of the scattering analyzer slab (red line in Fig. 4), and these were used to form intensity correlations as a function of translated incident field. These calculations were done for multiple randomly generated instances of the scattering analyzer and the incident field, allowing meaningful averages to be formed. In all cases, the fields conform well to zero-mean circular Gaussian statistics.

First, we simulated an analyzer with varying levels of scatter. Four types of analyzer scatter were considered: weak scatter type $\left(L_{y}=3 \mu \mathrm{m}, \epsilon_{r}=3\right)$, medium scatter type 1 $\left(L_{y}=3 \mu \mathrm{m}, \epsilon_{r}=5\right)$, medium scatter type $2\left(L_{y}=6 \mu \mathrm{m}\right.$, $\left.\epsilon_{r}=3\right)$, and heavy scatter type $\left(L_{y}=6 \mu \mathrm{m}, \epsilon_{r}=5\right)$. For each of these types, 2 slabs were randomly generated, and 10 random incident fields were simulated for each such slab. Figure 5(a) shows the influence of the analyzer's scatter level on intensity correlation, where more scatter resulted in more rapid decorrelation, as in the experimental data of Figs. 2 and 3. The error bars are $\pm \sigma_{e}$, where $\sigma_{e}$ is the standard error in estimating the mean resulting from the number of randomly generated incident fields (10), the number of randomly generated analyzer slabs (2), and the number of pairs of speckle intensity patterns for each field-analyzer pair (1-25, varies by shift distance).

Second, we considered varying the incident field's correlation length (speckle size), and the calculated decorrelation curves are plotted in Fig. 5(b) for a single slab type $\left(L_{y}=\right.$ $3 \mu \mathrm{m}, \epsilon_{r}=5$ ) and three different incident field correlation lengths. The correlation length was controlled by applying an ideal low-pass filter in the spectral domain to the (periodic) incident speckle field with different cutoffs, thereby regulating the amount of high-frequency content: large speckle size, $40 \pi / \Lambda$ (where $m \pi / \Lambda$ with $m=1$ is the fundamental periodic wave number in the $x$ direction); medium speckle size, 
$70 \pi / \Lambda$; and small speckle, with $140 \pi / \Lambda$. Figure $5(\mathrm{~b})$ shows that the calculated speckle intensity correlation as a function of the translated incident speckle field changed more slowly as the speckle size increased, which is consistent with the experimental result in Fig. 3. Simulations have thus confirmed our understanding of the influence of varying the amount of scattering in the analyzer and the incident speckle size on the spatial sensitivity to subwavelength motion.

While random scatter of light is limiting in some situations, it has been exploited in the concept of a random spectrometer, where the frequency-dependent transmission of a multimode optical fiber provides sensitivity in measured speckle to changes in frequency [17]. We provide a dimension for randomly scattering material by treating it as an analyzer for spatial changes in the incident field. One example application of the analyzer concept is as a lensless imager [18], which can utilize a thin layer of diffractive or diffusive material and a compressed-sensing-based computational inversion process to create a miniaturized, lens-free microscope. While increasing the thickness of the scattering analyzer layer would likely require a reconsideration of the image reconstruction processes involved [18,19], the potential exists for a substantial increase in the achievable spatial resolution. This work raises the prospect of regulating the statistical properties of a random medium to achieve high spatial sensitivity to changes in the incident field. For instance, aperiodic structures have field-control properties that are dependent on the specific features of the structure [20]. We now find a relationship between design rules that may be applied to create devices for a specific task and the general statistical character of a random medium acting as an analyzer for super-resolution spatial sensing. Finally, the analyzer is acting in a compressed sensing framework [21], where, in our case, spatial field information is encoded in speckle from multiply scattered light.

We have shown that the averaged speckle intensity correlation over subject movement decorrelates faster in the presence of a scattering analyzer, and that the more strongly scattering the analyzer, the faster the decorrelation. Consequently, with coherent light, it becomes possible to achieve far-subwavelength far-field spatial resolution, as dictated by the associated noise processes. This super-resolution remote sensing approach with a randomly scattering analyzer and a fixed detector aperture is applicable to all wave types, and hence offers substantial scope for impact in the physical sciences. If multiple dimensions of movement were to be incorporated (e.g., both $\Delta x$ and $\Delta y$ ), prior information would be required in order to determine this directional information, since any change in the field can impact the measured intensity. Even without knowing the direction in which the system has changed, however, we can still infer useful information, such as the extent of the translation or the frequency of vibration. The speckle field could change due to motion of a small number of scatterers [22], and insight beyond a statistical theory related to intensity correlations over object position [23] could be provided by an eigenmode (or similar) analysis of the transmission matrix [2] (T, such as with a plane-wave decomposition) of the resolution-enhancing analyzer presented in this work, along the lines of a previous waveguide treatment [6]. The theoretical underpinnings of access to subwavelength far-field spatial information with motion and an analyzer can be obtained from the distributions of the singular values (the eigenvalues) of $\mathbf{T}^{H} \mathbf{T}$, with $\mathbf{T}^{H}$ the Hermitian (complex conjugate) transpose, which transition from quarter-circle-like (Wigner [5]) to being increasingly concentrated around zero (and more like the bimodal distribution that has been previously described [2,24-26]), as the level of scatter increases in the analyzer. Using multiple wavelengths may unlock additional information, and therefore increased sensitivity [27]. While we have treated the informational aspects of the analyzer, achieving identification of an object of interest is possible with calibration [28]. Under a constraint set, it may also be possible to image at this subwavelength length scale, as proposed using the concept of motion in structured illumination [23,29], but now with relative motion of a field (perhaps scattered from an object) on the other side of a random analyzer with the specific purpose of enhancing spatial information. The analyzer concept also aligns well with that of optical physical unclonable functions [30], and the analyzer slab properties explored in this work could help to increase security in the quantum photonics domain. Opportunities with partially coherent light [31] and speckle-based astronomical imaging [32] may also be enabled.

This work was supported by the National Science Foundation (NSF) under Grants No. 1610068 and No. 1618908, and by the Air Force Office of Scientific Research under Grant No. FA9550-19-1-0067.
[1] A. Goetschy and A. D. Stone, Filtering Random Matrices: The Effect of Incomplete Channel Control in Multiple Scattering, Phys. Rev. Lett. 111, 063901 (2013).

[2] C. W. J. Beenakker, Random-matrix theory of quantum transport, Rev. Mod. Phys. 69, 731 (1997).

[3] S. Rotter and S. Gigan, Light fields in complex media: Mesoscopic scattering meets wave control, Rev. Mod. Phys. 89, 015005 (2017).

[4] Z. Shi and A. Z. Genack, Transmission Eigenvalues and the Bare Conductance in the Crossover to Anderson Localization, Phys. Rev. Lett. 108, 043901 (2012).
[5] E. P. Wigner, On the distribution of the roots of certain symmetric matrices, Ann. Math. 67, 325 (1958).

[6] N. Bender, A. Yamilov, H. Y1lmaz, and H. Cao, Fluctuations and Correlations of Transmission Eigenchannels in Diffusive Media, Phys. Rev. Lett. 125, 165901 (2020).

[7] S. M. Popoff, G. Lerosey, R. Carminati, M. Fink, A. C. Boccara, and S. Gigan, Measuring the Transmission Matrix in Optics: An Approach to the Study and Control of Light Propagation in Disordered Media, Phys. Rev. Lett. 104, 100601 (2010).

[8] H. Yu, T. R. Hillman, W. Choi, J. O. Lee, M. S. Feld, R. R. Dasari, and Y. K. Park, Measuring Large Optical Transmission 
Matrices of Disordered Media, Phys. Rev. Lett. 111, 153902 (2013).

[9] E. Betzig, G. H. Patterson, R. Ssvougrat, O. W. Lindwasser, S. Olenych, J. S. Bonifacino, M. W. Davidson, J. LippincottSchwartz, and H. F. Hess, Imaging intracellular fluorescent proteins at nanometer resolution, Science 313, 1642 (2006).

[10] S. T. Hess, T. P. Girirajan, and M. D. Mason, Ultra-high resolution imaging by fluorescence photoactivation localization microscopy, Biophys. J. 91, 4258 (2006).

[11] M. J. Rust, M. Bates, and X. Zhuang, Sub-diffractionlimit imaging by stochastic optical reconstruction microscopy (STORM), Nat. Methods 3, 793 (2006).

[12] S. Oppel, T. Büttner, P. Kok, and J. von Zanthier, Superresolving Multiphoton Interferences with Independent Light Sources, Phys. Rev. Lett. 109, 233603 (2012).

[13] A. Classen, J. von Zanthier, M. O. Scully, and G. S. Agarwal, Superresolution via structured illumination quantum correlation microscopy, Optica 4, 580 (2017).

[14] O. Schwartz and D. Oron, Improved resolution in fluorescence microscopy using quantum correlations, Phys. Rev. A 85, 033812 (2012).

[15] D. Gatto Monticone, K. Katamadze, P. Traina, E. Moreva, J. Forneris, I. Ruo-Berchera, P. Olivero, I. P. Degiovanni, G. Brida, and M. Genovese, Beating the Abbe Diffraction Limit in Confocal Microscopy via Nonclassical Photon Statistics, Phys. Rev. Lett. 113, 143602 (2014).

[16] Q. Luo and K. J. Webb, Parametrization of speckle intensity correlations over object position for coherent sensing and imaging in heavily scattering random media, Phys. Rev. Research 2 , 033148 (2020).

[17] P. Ambichl, W. Xiong, Y. Bromberg, B. Redding, H. Cao, and S. Rotter, Super- and Anti-Principal-Modes in Multimode Waveguides, Phys. Rev. X 7, 041053 (2017).

[18] N. Antipa, G. Kuo, R. Heckel, B. Mildenhall, E. Bostan, R. $\mathrm{Ng}$, and L. Waller, Diffusercam: Lensless single-exposure 3D imaging, Optica 5, 1 (2018).

[19] G. Kuo, F. L. Liu, I. Grossrubatscher, R. Ng, and L. Waller, Onchip fluorescence microscopy with a random microlens diffuser, Opt. Express 28, 8384 (2020).
[20] Y.-C. Hsueh and K. J. Webb, Electromagnetic field control with binary aperiodic nanostructures, J. Opt. Soc. Am. B 34, 2059 (2017).

[21] D. L. Donoho, Compressed sensing, IEEE Trans. Inf. Theory 52, 1289 (2006).

[22] R. Berkovits, Sensitivity of the multiple-scattering speckle pattern to the motion of a single scatterer, Phys. Rev. B 43, 8638 (1991).

[23] K. J. Webb and Q. Luo, Theory of speckle intensity correlations over object position in a heavily scattering random medium, Phys. Rev. A 101, 063827 (2020).

[24] O. Dorokhov, On the coexistence of localized and extended electronic states in the metallic phase, Solid State Commun. 51, 381 (1984).

[25] Y. Imry, Active transmission channels and universal conductance fluctuations, Europhys. Lett. 1, 249 (1986).

[26] J. B. Pendry, A. MacKinnon, and P. Roberts, Universality classes and fluctuations in disordered systems, Proc. R. Soc. Lond. A 437, 67 (1992).

[27] M. A. Webster, K. J. Webb, and A. M. Weiner, Temporal Response of a Random Medium from Third-Order Laser Speckle Frequency Correlations, Phys. Rev. Lett. 88, 033901 (2002).

[28] J. A. Newman, Q. Luo, and K. J. Webb, Imaging Hidden Objects with Spatial Speckle Intensity Correlations over Object Position, Phys. Rev. Lett. 116, 073902 (2016).

[29] K. J. Webb, Y. Chen, and T. A. Smith, Object Motion with Structured Optical Illumination as a Basis for FarSubwavelength Resolution, Phys. Rev. Applied 6, 024020 (2016).

[30] S. A. Goorden, M. Horstmann, A. P. Mosk, B. Škorić, and P. W. Pinkse, Quantum-secure authentication of a physical unclonable key, Optica 1, 421 (2014).

[31] R. Carminati, G. Cwilich, L. S. Froufe-Pérez, and J. J. Sáenz, Speckle fluctuations resolve the interdistance between incoherent point sources in complex media, Phys. Rev. A 91, 023807 (2015).

[32] A. Labeyrie, Attainment of diffraction limited resolution in large telescopes by Fourier analysing speckle patterns in star images, Astron. Astrophys. 6, 85 (1970). 